\title{
Peptide-centric approaches provide an alternative perspective to re-examine quantitative proteomic data
}

\author{
Zhibin Ning ${ }^{\dagger}$, Xu Zhang ${ }^{\dagger}$, Janice Mayne, Daniel Figeys ${ }^{*}$ \\ Ottawa Institute of Systems Biology, Department of Biochemistry, Microbiology and Immunology, \\ University of Ottawa, 451 Smyth Rd., Ottawa, ON, Canada, K1H8M5 \\ ${ }^{\dagger}$ Contributed equally \\ *To whom correspondence should be addressed: Daniel Figeys \\ Phone: 613-562-5800 ext 8674. \\ Fax: 613-562-5655. \\ E-mail: dfigeys@uottawa.ca
}

\section{Abstract}

Quantitative proteomics can provide rich information on changes in biological functions and processes. However, its accuracy is affected by the inherent information degeneration found in bottom-up proteomics. Therefore, the precise protein inference from identified peptides can be mistaken since an ad hoc rule is used for generating a list of protein groups that depends on both the sample type and the sampling depth. Herein, we propose an alternative approach for examining quantitative proteomic data which is peptide-centric instead of protein-centric. We discuss the feasibility of the peptide-centric approach which was tested on several quantitative proteomic datasets. We show that peptide-centric quantification has several advantages over protein level analysis: (1) it is more sensitive for sample segregation, (2) it avoids the issues associated with protein inference, (3) and it can retrieve significant peptides lost in protein-centric quantification for further downstream analysis. 


\section{Supplemental Figures}

\section{Figure S1}

Distribution of distinct peptides per protein from large scale data. Data come from reference ${ }^{1}$. Even larger scale statistic is described in reference ${ }^{2,3}$.

\section{Distribution of Peptides per Protein}

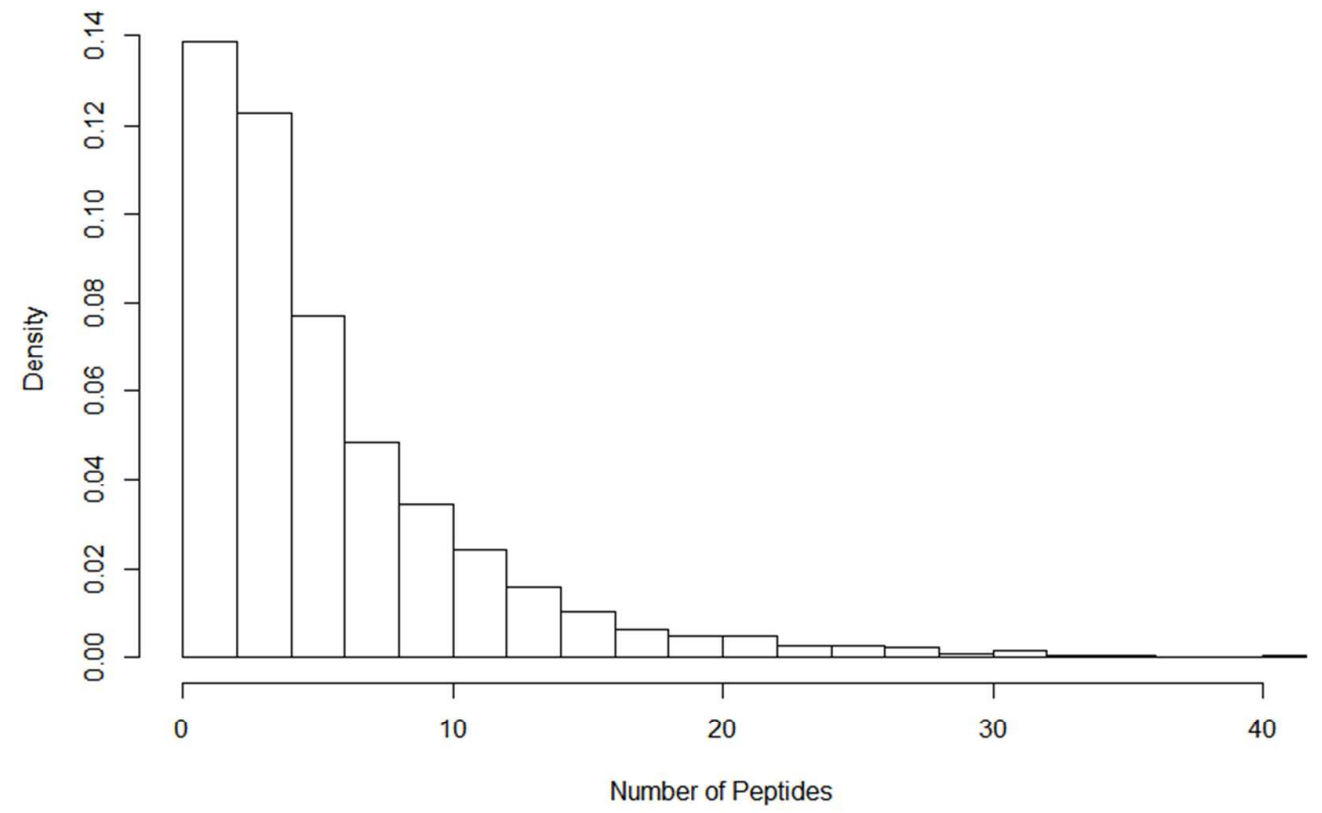

\section{Figure S2}

Protein quantification is contributed from the same set of identified proteins in theory (upper panel) or different subsets of peptide in practice (lower panel).

Sample A Sample B Sample C Sample D Sample E

Protein $\mathrm{X}$

Ideally:

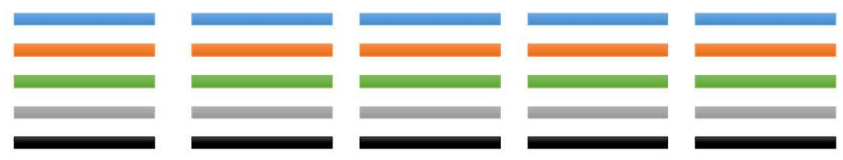

Protein X In reality:

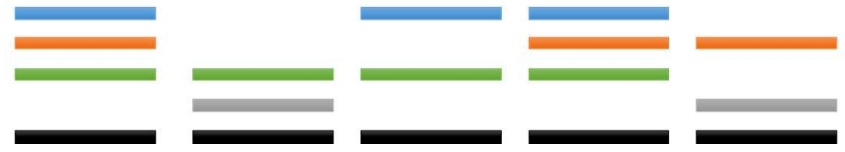




\section{Figure S3}

Discrepancy between protein and peptide in quantification dynamic range. A: Quantification dynamic range of proteins and corresponding peptides in one sample; B: A zoom-in of the left region in A. C: Quantification dynamic range of proteins and corresponding peptide across different samples. Quantification data from reference ${ }^{1}$.

A:

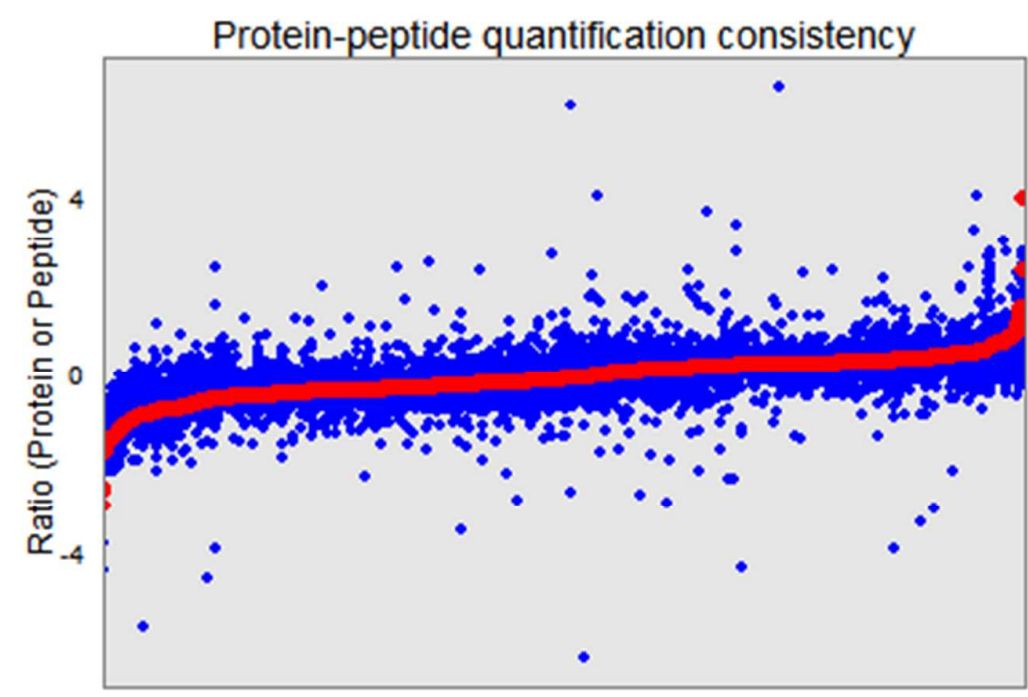

Protein Groups

B:

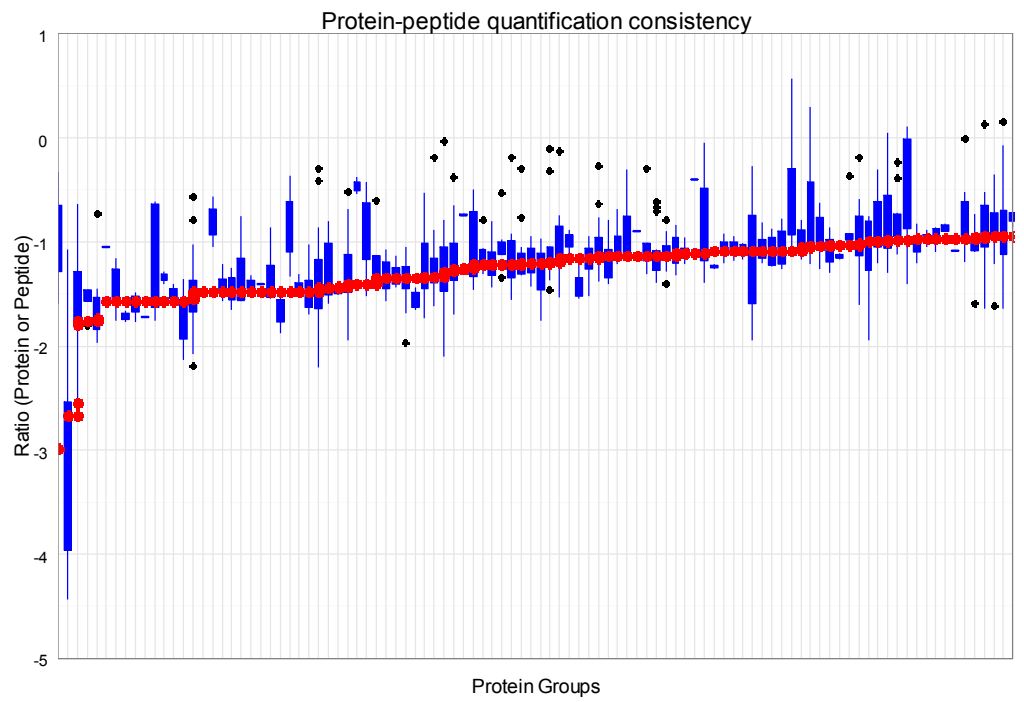

C: 


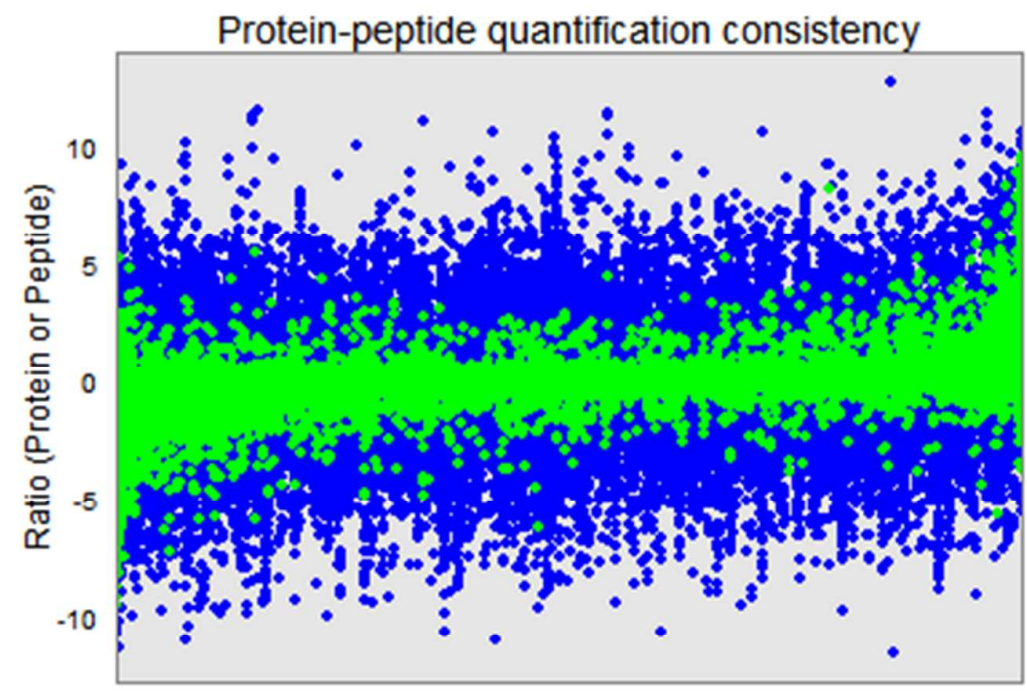

Protein Groups

\section{Figure S4}

An example of significantly changed peptide discarded by protein-centric quantification analysis, Protein show here did not pass the test as circadian rhythmic. Average log2-tranformed $\mathrm{L} / \mathrm{H}$ ratios of 3 replicates in each time points were shown.

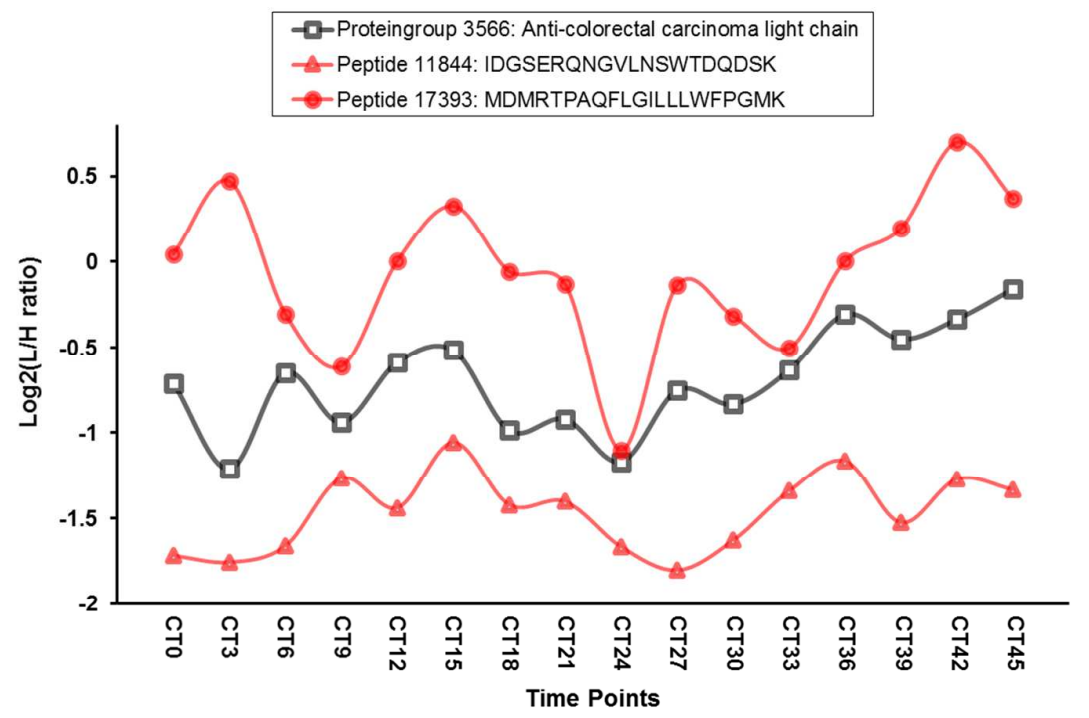




\section{Material and Method:}

\section{Analysis of diffuse Large B-cell lymphoma subtypes dataset}

Raw data and MaxQuant output for diffuse large B-cell lymphoma subtypes were downloaded from ProteomeXchange Consortium at PXD002098. Normalized L/H ratios of protein groups or peptides were used for the analysis, and only those with valid value in all the samples were included for further analysis, which was same with the original paper in Figure $4 A^{4}$. Principal component analysis (PCA) was carried out in Matlab (version 2014b, MathWorks Inc.) using princomp function, and the first and second principal component scores were plotted in Matlab.

\section{Analysis of gut metaproteomic dataset}

Gut metaproteomic data were collected from a small cohort of inflammatory bowel disease (IBD) patients including 5 control individuals, 5 Crohn's disease (CD) and 4 ulcerative colitis (UC) patients. Briefly, the intestinal aspirate samples were collected from distal colon through colonscope as per previously published protocol ${ }^{5}$. Bacterial cells were then harvested by differential centrifugation and subjected for protein extraction and in-solution trypsin digestion. The tryptic peptides were desalted for mass spectrometry analysis on LTQ Orbitrap Velos Elite (Thermo Fisher Scientific, San Jose, CA). Maxquant (Version 1.3.0.5) was used for peptide identification and label-free protein quantification by using an in-house intestinal bacterial protein database. The following parameters were used: cysteine carbamidomethylation was selected as the fixed modification; and the methionine oxidation and protein $\mathrm{N}$-terminal acetylation were set variable modifications. Enzyme specificity was set to trypsin/P. Up to two missing cleavages of were allowed. The precursor ion mass tolerances were $10 \mathrm{ppm}$, and fragment ion mass tolerance was 0.6 Da for MS/MS spectra. The false discovery rate (FDR) for peptide and protein was set at $1 \%$ with a 
minimum peptide length of six. The LFQ intensity of proteingroups and peptides in MaxQuant search results were imported into Perseus for data preprocessing and filtering. Proteingroups with valid values in at least half of the samples were kept for further analysis and the missing values were imputed with nearest-neighbor method in Matlab version 2014b using knnimpute function ${ }^{6}$. Principal component analysis (PCA) was also carried out in Matlab using princomp function, and the first and second principal component scores were plotted in Matlab.

\section{Analysis of the mouse liver and SCN circadian datasets}

Raw data and Maxquant output for Chiang's dataset ${ }^{7}$ were directly downloaded from the ProteomeXchange Consortium at PXD000778. Raw L/H ratios from protein group and modified peptide files were used and only those with valid value in at least

half of the samples were included for further JTK analysis ${ }^{8}$. A prior imputation of missing value was made by using nearest-neighbor method. For JTK analysis, a circadian cycle of $12 \mathrm{~h}$ to $24 \mathrm{~h}$ were tested for all of the protein groups or modified peptides. Those with ADJ.P value less than 0.05 were considered as circadian proteins or peptides ${ }^{8}$.

Raw data and Maxquant output for Robles's dataset ${ }^{1}$ were downloaded from ProteomeXchange Consortium AT PXD000601. Normalized L/H ratios of protein groups or peptides were used for the analysis, and only those with valid value in at least half of the samples were included for further analysis. As described above, nearest-neighbor method was used for missing value imputation. For JTK analysis, a circadian cycle of $12 \mathrm{~h}$ to $36 \mathrm{~h}$ were tested for all of the protein groups or peptides. Those with ADJ.P value less than 0.05 were considered as circadian proteins or peptides. 


\section{Reference:}

(1) Robles, M. S.; Cox, J.; Mann, M. PLoS Genet 2014, 10, e1004047.

(2) Kim, M. S.; Pinto, S. M.; Getnet, D.; Nirujogi, R. S.; Manda, S. S.; Chaerkady, R.; Madugundu, A. K.; Kelkar, D. S.; Isserlin, R.; Jain, S.; Thomas, J. K.; Muthusamy, B.; Leal-Rojas, P.; Kumar, P.; Sahasrabuddhe, N. A.; Balakrishnan, L.; Advani, J.; George, B.; Renuse, S.; Selvan, L. D.; Patil, A. H.; Nanjappa, V.; Radhakrishnan, A.; Prasad, S.; Subbannayya, T.; Raju, R.; Kumar, M.; Sreenivasamurthy, S. K.; Marimuthu, A.; Sathe, G. J.; Chavan, S.; Datta, K. K.; Subbannayya, Y.; Sahu, A.; Yelamanchi, S. D.; Jayaram, S.; Rajagopalan, P.; Sharma, J.; Murthy, K. R.; Syed, N.; Goel, R.; Khan, A. A.; Ahmad, S.; Dey, G.; Mudgal, K.; Chatterjee, A.; Huang, T. C.; Zhong, J.; Wu, X.; Shaw, P. G.; Freed, D.; Zahari, M. S.; Mukherjee, K. K.; Shankar, S.; Mahadevan, A.; Lam, H.; Mitchell, C. J.; Shankar, S. K.; Satishchandra, P.; Schroeder, J. T.; Sirdeshmukh, R.; Maitra, A.; Leach, S. D.; Drake, C. G.; Halushka, M. K.; Prasad, T. S.; Hruban, R. H.; Kerr, C. L.; Bader, G. D.; lacobuzio-Donahue, C. A.; Gowda, H.; Pandey, A. Nature 2014, 509, 575-581.

(3) Wilhelm, M.; Schlegl, J.; Hahne, H.; Moghaddas Gholami, A.; Lieberenz, M.; Savitski, M. M.; Ziegler, E.; Butzmann, L.; Gessulat, S.; Marx, H.; Mathieson, T.; Lemeer, S.; Schnatbaum, K.; Reimer, U.; Wenschuh, H.; Mollenhauer, M.; Slotta-Huspenina, J.; Boese, J. H.; Bantscheff, M.; Gerstmair, A.; Faerber, F.; Kuster, B. Nature 2014, 509, 582-587.

(4) Deeb, S. J.; D'Souza, R. C.; Cox, J.; Schmidt-Supprian, M.; Mann, M. Mol Cell Proteomics 2012, 11, 77-89.

(5) Jimenez-Rivera, C.; Haas, D.; Boland, M.; Barkey, J. L.; Mack, D. R. Gastroenterology research and practice 2009, 2009, 518932.

(6) Troyanskaya, O.; Cantor, M.; Sherlock, G.; Brown, P.; Hastie, T.; Tibshirani, R.; Botstein, D.; Altman, R. B. Bioinformatics 2001, 17, 520-525.

(7) Chiang, C. K.; Mehta, N.; Patel, A.; Zhang, P.; Ning, Z.; Mayne, J.; Sun, W. Y.; Cheng, H. Y.; Figeys, D. PLoS Genet 2014, 10, e1004695.

(8) Hughes, M. E.; Hogenesch, J. B.; Kornacker, K. Journal of biological rhythms 2010, 25, 372-380. 